\title{
Evidence vs. efficacy in allergen- specific immunotherapy: Considerations using the example of tradable products in Germany
}

Randolf Brehler ${ }^{1}$, Ludger Klimek ${ }^{2}$, Christian Vogelberg ${ }^{3}$, Thomas Werfel ${ }^{4}$, Oliver PfaAr ${ }^{2,}{ }^{5}$, Eckard Hamelmann ${ }^{6,7}$

'Outpatient Allergology Clinic, Occupational Dermatology and Environmental Medicine, Department of Dermatology, Münster University Hospital, Münster, Germany; ${ }^{2}$ Center for Rhinology and Allergology, Wiesbaden, Germany; ${ }^{3}$ Department of Pediatrics and Adolescent Medicine, Carl Gustav Carus University Hospital, Dresden, Germany; ${ }^{4}$ Department of Dermatology, Venereology, and Allergology, Hannover Medical University, Hannover, Germany; ${ }^{5}$ ENT at Mannheim University Hospital, Mannheim, Germany; ${ }^{6}$ Ruhr Allergy Center, Ruhr University Bochum, Germany; ${ }^{7}$ Bethel Pediatric Center, Bielefeld, Germany

Key words hypersensitization - allergic rhinoconjunctivitis allergology guideline - evidence - efficacy

Submitted

December 7, 2015

\section{Accepted}

January 26, 2016

German version www.springermedizin.de/ allergo-journal

\section{Abstract}

The recently published S2k-guideline on (allergen-) specific immunotherapy (AIT) provides an excellent overview of the evidence on allergen preparations available for AIT in Germany based on the published efficacy studies. Publications based on the guideline are currently being used by the German associations of statutory health insurance physicians and German health insurance funds to open a discussion on the reimbursement status of allergen preparations. In our view, calling the reimbursement status of perscribable and tradable AIT preparations into question on the basis of an assessment of the current body of evidence in the guideline is to be rigidly opposed. In Germany the Paul Ehrlich Institute (PEI) is the only authority

\section{Evidence and efficacy}

(Allergen-)Specific immunotherapy (AIT) is currently the only treatment available that is capable of inducing specific tolerance to individual allergens, particularly in allergies to inhalant allergens [1]. In principle, due to its disease-modifying effects that may manifest as asthma prevention and the prevention of new sensitizations, AIT is superior to purely symptomatic treatment [2, 3]. However, studies have proven these effects for only a scant number of products. A variety of preparations are available for subcutaneous and sublingual use, whereby native or chemically modified allergens (allergoids) can be used. The practitioner needs to establish which empowered to decide on the marketability of AIT preparations, and decisions on the reimbursement status of AIT products need to be based on costbenefit analyses and not solely on an evaluation of the evidence. The present article aims to examine the relationship between the evidence, efficacy, tradability, and reimbursability of AIT preparations.

Cite this as Brehler R, Klimek L, Vogelberg C, Werfel T, Pfaar O, Hamelmann E. Evidence vs. efficacy in allergen-specific immunotherapy: Considerations using the example of tradable products in Germany. Allergo J Int 2016; 25:38-43

DOI: 10.1007/s40629-016-0096-2

product should be used, taking into consideration efficacy, safety, time expenditure for the patient, as wells as costs.

The revised S2k-guideline [4] published at the end of 2014 is based on the current state of knowledge and provides physicians with excellent support when deciding for or against AIT, as well as in the selection of allergen preparations. One of the guideline's core statements is a call to evaluate each individual preparation on the basis of the study results available for that preparation, irrespective of its mode of administration. In the opinion of the authors, approved allergen preparations with documented efficacy and safety, or preparations mar- 
ketable under the Therapy Allergen Ordinance (TAO) for which efficacy and safety have already been documented in clinical trials meeting World Allergy Organization (WAO) or European Medicines Agency (EMA) standards, „should be preferentially used" [4].

AIT products are essentially challenging to compare due to their heterogeneous composition. To date, the characterization and standardization of allergen extracts has been performed according to manufacturer-specific standards and, even when major allergen concentrations are given, these do not permit conclusions to be drawn on possible efficacy, since methods for the analysis of major allergens are not consistent, and most allergen extracts contain several major allergens. This has lead to the indispensable requirement (as with other drugs) for reliable studies to analyze each individual AIT preparation for efficacy and safety. This presents the prescribing physician with the dilemma of needing to be familiar with, and capable of evaluating, the study data.

By way of support here, the guideline refers readers to tables listing allergen preparations that fall under the German TOA. These tables are not an integral part of the guideline. They provide information on the following:

- Current marketing authorization status of individual products

$\begin{array}{ll}\text { Abbreviations } \\ \text { AIT } & \text { (Allergen-)specific immunotherapy } \\ \text { AMG } & \begin{array}{l}\text { Medicinal Products Act } \\ \text { (Arzneimittelgesetz) }\end{array} \\ \text { DDD } & \text { Defined daily dose } \\ \text { EMA } & \text { European Medicines Agency } \\ \text { G-BA } & \text { Joint Federal Committee (Gemeinsamer } \\ & \text { Bundesausschuss) } \\ \text { ITT } & \text { Intent to treat } \\ \text { IQWIG } & \text { Institute for Quality and Efficiency in } \\ & \begin{array}{l}\text { Health Care (Institut für Qualität und } \\ \text { Wirtschaftlichkeit im Gesundheitswesen) }\end{array} \\ \text { PP } & \text { Per protocol } \\ \text { QUALY } & \text { Quality-adjusted life years } \\ \text { SCIT } & \text { Subcutaneous immunotherapy } \\ \text { SGB } & \text { German Social Code (Sozialgesetzbuch) } \\ \text { SLIT } & \text { Sublingual immunotherapy } \\ \text { TAO } & \text { Therapy Allergen Ordinance } \\ \text { WHO } & \text { World Health Organization }\end{array}$

_ The number of full publications evidencing the efficacy of each individual preparation listed - An overview of trials that have been conducted with official approval (according to the EMA register www.clinicaltrialsregister.eu [www.dgaki. de/leitlinien/s2k-leitlinie-sit/clinical-trials-register-eu-nachzulassungen und www.dgaki.de/ leitlinien/s2k-leitlinie-sit/clinical-trials-register-eu-neuzulassungen])

The tables are updated bi-annually and brought in line with the current status. Only the results of clinical trials that fulfill conditions defined on the basis of WAO criteria are included in the list (www. dgaki.de/leitlinien/s2k-leitlinie-sit/sit-produk-

te-studien-zulassung) [5].

The conditions to be fulfilled for the guideline are the following:

1. Standardized allergen extract with dose information,

2. randomized, double-blind, placebo-controlled study design,

3.information relating to a combined symptom-medication score and/or the two individual scores,

4. information relating to statistical analysis and statistically significant results,

5. a threshold in effcacy of $20 \%$ above placebo. The first point represents a minimum requirement in terms of product characterization stipulated by the Paul Ehrlich Institute for the marketing authorization of an AIT product. Requirements 2-4 emphasize the evidence documenting that the product is effective. The term evidence (see definition in Tab. 1) stems from the Latin "evidentia“ and is the colloquial term for "obviousness" meaning that something does not need to be questioned [6]. Thus, evidence does not assess the magnitude of efficacy, but concerns rather the reliability with which something is proven to be effective.

The fifth criterion specifies a minimum level of efficacy. This criterion is not relevant to the marketing authorization of an AIT product at the Paul Ehrlich Institute. The requirement is based on the concept that specific immunotherapy that is far more costly than pharmacotherapy needs to demonstrate at least an efficacy comparable to that of (here in the sense of antihistamine and/or topical corticosteroids treatment). However, it must be borne in mind that clinical studies to assess the effect of specific immunotherapy or of pharmacotherapy are designed differently, and that the possible disease-modifying effect of AIT is not taken into account in this comparison. According to published studies, a $20 \%$ improvement in the symptom and medication score (generally measured in the first year following AIT initiation) results in a relevant improvement in quality of life. 
When considering the various AIT studies, one should bear in mind that the resulting strength of treatment can be affected by a number of factors that, to a certain extent, cannot be influenced and which are independent of the investigated preparation:

_-Symptom severity in the study population

_Extent of sensitization among subjects

- Degree of pollination during the study season

—Definition of the endpoint

_ Statistical analysis

A further crucial factor is whether the intent-totreat (ITT) or the per-protocol (PP) population (see definitions in Tab. 1) is analyzed. While effect sizes may be identical in the PP and ITT, they can also differ widely.

A harmonization of studies is sought on both an international and a national level. However, the efficacy of different allergen products can only be compared if different products are directly used in the same study. At present, it appears highly unlikely that head-to-head studies of this kind will be conducted in the foreseeable future.

Evidence assesses the reliability with which an intervention is shown to be effective and does not permit conclusions to be drawn on the size of the intervention's effect. Thus, a lack of evidence is not tan-

\begin{tabular}{|l|l|l|l|l|l|l|}
\hline Tab. 1: Definitions & Definition \\
\hline Eerm & $\begin{array}{l}\text { Efficacy compared with placebo as the difference } \\
\text { between the placebo and verum group in terms of } \\
\text { defined efficacy parameters }\end{array}$ \\
\hline Evidence & $\begin{array}{l}\text { Stems from } \text {,evidentia"; reliability of the evidence of } \\
\text { efficacy }\end{array}$ \\
\hline Evidence-based medicine & $\begin{array}{l}\text { The requirement in medical treatment to make a } \\
\text { patient-oriented decision, where possible, on the basis } \\
\text { of empirically proven efficacy }\end{array}$ \\
\hline Per-protocol analysis (PP) & $\begin{array}{l}\text { Analysis of data from patients actually treated according } \\
\text { to the protocol }\end{array}$ \\
\hline $\begin{array}{l}\text { Intention-to-treat analysis } \\
\text { (ITT) }\end{array}$ & $\begin{array}{l}\text { Data of patients in the verum or placebo group are } \\
\text { analyzed, irrespective of whether treatment was subse- } \\
\text { quently carried out according to protocol }\end{array}$ \\
\hline Tradability & \begin{tabular}{l} 
Prerequisite for a drug to be sold and used \\
\hline Reimbursability
\end{tabular} & $\begin{array}{l}\text { The Joint Federal Committee holds the authority to } \\
\text { decide on limiting or excluding the performance and } \\
\text { prescription of services }\end{array}$ \\
\hline Finished medicinal products & $\begin{array}{l}\text { Finished medicinal products are medicinal products that } \\
\text { are manufactured in advance and placed on the market } \\
\text { in packaging intended for the consumer, or other medi- } \\
\text { cinal products intended for consumers, the preparation } \\
\text { of which involves an industrial procedure, or which are } \\
\text { commercially manufactured (except in pharmacies) }\end{array}$ \\
\hline
\end{tabular}

tamount to a lack of efficacy, and the intervention with the highest level of evidence is not necessarily the most effective. However, a basic requirement should be that efficacy is shown in controlled studies for all preparations containing common allergens currently on the market (see below). The TAO defines the trial requirements that need to be fulfilled for marketing authorization.

\section{Marketing authorization}

AIT preparations that were granted marketing authorization by the relevant authorities before the TAO came into force [8] have proven their efficacy in studies that satisfied the marketing authorization requirements at that time. According to the TAO, these preparations are not required to be investigated in new studies fulfilling current standards; they remain permanently tradable. Although this fact may appear worthy of discussion, it is understandable given that marketing authorization of drugs cannot be withdrawn simply because the requirements made of trials to establish efficacy have changed. Since the trials conducted in the past partially failed to satisfy WAO requirements for inclusion in the guideline tables, the tables contain older authorized products in particular, for which no efficacy trials are available (since the published trials do not fulfill current criteria). However, this lack of data does not imply that no trials exist or even that these preparations are necessarily ineffective. More importantly, this is in no way linked to the assertion that the marketing authorization of these preparations is questionable and that their prescription represents grounds for recourse. Thus, the guideline does not release allergologists from their duty to assess trial data on the products they use on an individual basis.

\section{AIT with rare allergens}

All the above-mentioned considerations apply only to AIT using frequently occurring allergens that fall under the TAO (species from the Poaceae family, excluding Poa mays [grasses, excluding maize], Betula sp. [species in the birch genus], Alnus sp. [species in the alder genus], Corylus sp. [species of the hazel genus], Dermatophagoides sp. [species in the house dust mite genus], bee venom, and wasp venom) [8]. Despite the TAO's entry into force, current legislation continues to make provision for the option to prescribe individual formulations of all other therapy allergens not belonging to the above-mentioned group (for instance, weed pollen [e.g., ragweed, mugwort, plantain, etc.], mold [e.g., Alternaria, Aspergillus, Cladosporium], storage mites, animal dander [e.g., cat]).

The legislature chose this path for good reason. The studies involving large patient numbers that would be needed for the approval of rarer allergens 
are virtually impracticable due to the number of patients required. Nevertheless, patients with allergies to rare allergens are equally entitled to cause-oriented treatment using AIT. Preparations produced and marketed according to $\$ 21$ paragraph 2,1 b of the Medicinal Products Act (Arzneimittelgesetz, AMG) of the Federal Republic of Germany continue to be available to these patients as individual formulations for AIT. The 14th amendment of the AMG of 2005 regulates the exemption of therapy allergens produced for individual patients from licensing requirements. Specific immunotherapeutic agents produced on the basis of a prescription formulation are explicitly exempted from the obligation to obtain marketing authorization in $\$ 21$ paragraph 2 , $1 \mathrm{~b}$ of the AMG: "A marketing authorisation ( $\mathrm{Zu}$ lassung) shall not be required for medicinal products which ... are prepared on prescription for individual persons." Here again, no grounds for recourse are provided for.

\section{Reimbursement status}

There has been a discussion in recent months on possible restrictions on the reimbursment of allergen extracts used for AIT. One publication in particular [9], which virtually compiles a positive list of products for AIT, forms the basis for this discussion. The publication categorizes allergen products into a pyramid according to "first choice" to "last choice." While the current tables in the guideline appendix have our full endorsement, we see a real hazard in terms of interpretation posed by the specific classification of products - conveyed as the personal recommendation of the publication's author by virtue of the pyramid structure - into "recommended" and "not recommended", which does not lie in the intention of the guideline. In our view, representing products in such a way bears the risk that healthcare actors could indirectly instrumentalize the guideline as a means to decide on the reimbursability of preparations. The guideline, as well as the tables in the appendix explicitly state that the table is "not suited as a positive or negative list for decision-making on prescribability or reimbursability." It is our opinion that deriving a positive list based on the guideline tables is also particularly unjustified since the issue does not relate to the actual efficacy of AIT products, but rather to merely presenting evidence according to criteria based on WAO recommendations. It is currently not possible to conduct an accurate, scientifically founded comparative assessment of the efficacy of the various AIT products, meaning that the prescriber of these products must not be limited in his therapeutic freedom via the loophole of decisions on reimbursability. However, for patients' well-being, any prescription of an AIT preparation should be based on efficacy data, and it is the responsibility of the prescribing physician to establish whether studies have proven the efficacy of the preparation that he/she wishes to prescribe.

\section{Cost-benefit analysis}

Whereas the Paul Ehrlich Institute decides on the marketability of drugs, guidelines aimed at ensuring medical care - as compiled by the Joint Federal Committe according to $\$ 92$ of the German Social Code (Sozialgesetzbuch, SGB) V - should enable the physician to make a cost-effective and appropriate selection of drug therapy. It has been specified that information shall be included that permits the physician to make an appropriate treatment and drug selection that is also based on treatment benefit in relation to treatment costs, thereby serving the cost effectiveness of the prescription. According to $₫ 35 \mathrm{~b}$ SGB V, the Joint Committee tasks the German Institute for Quality and Efficiency in Health Care (Institut für Qualität und Wirtschaftlichkeit im Gesundheitswesen, IQWIG) with drawing up a cost-benefit analysis in which both the benefit to the patient (disease improvement, reduced disease duration, increased life expectancy, reduced side effects, improved quality of life) and the economic analysis (appropriateness and reasonableness of requesting the insured community to meets the costs) need to be taken into consideration. Thus, a well-founded selection of reimbursable or non-reimbursable products must be made on the basis of cost-benefit analyses. A valid, preparation-specific calculation of the benefit conferred by AIT is currently not possible since, in addition to reduced medication use and improved ability to work, as well as the long-term effects in terms of asthma reduction and the prevention of new sensitizations, treatment with each individual preparation needs to be assessable.

Calculating the cost of AIT is also not unproblematic. Due to varying treatment protocols, calculating defined daily doses (DDD) is not helpful and is considered obsolete today. Calculating costs over a defined period of time (the costs of treatment over a 3-year period are often used) is favored. However, it should be borne in mind here that the standard period for subcutaneous immunotherapy (SCIT) is currently 3 years, while a period of $3-5$ years is increasingly favored for preparations used in the context of sublingual immunotherapy (SLIT). There is a discussion as to whether a single treatment cycle of several weeks for peptide therapy may be adequate. Therefore, rightfully speaking, the costs of a „complete treatment cycle" need to be assumed as a basis for calculating costs.

Alternatively, comparative models in which the financial expense of achieving a predefined outcome 
or quality-adjusted life years (QUALY) using different treatments are under discussion. However, studies of this kind are still awaited for the individual allergen preparations.

\section{Conclusion}

The guideline provides an excellent overview of the AIT preparations used in Germany and, with its tables, refers the reader to a current overview of the marketing approval status of these products, the published study results that fulfill today's criteria (according to WAO), as well as the official approval to conduct AIT trials. Using the guideline to deduce the reimbursability of allergen products is to be rigorously rejected. Reimbursability needs to be based on cost-benefit calculations, which are currently not practicable in a preparation-specific manner. The fact that it was not the intention of the guideline authors to influence the reimbursability of AIT products is clear from the wording in the tables: „The tables are not suitable as a basis for decision-making on prescribability or reimbursability in the sense of a positive or negative list."

Prof. Dr. Randolf Brehler

Outpatient Allergology Clinic, Occupational

Dermatology and Environmental Medicine

Department of Dermatology

University Hospital Münster

Von-Esmarch-Straße 58

48149 Münster, Germany

E-Mail: r.brehler@uni-muenster.de

\section{Comment}

A number of passages in the present article have been taken from a statement issued by the three specialist allergology societies, AeDA, DGAKI, and GPA, and published in Allergo Journal on the prescription forum No. $34 / 2015$ of the German association of statutory health insurance physicians (Baden-Württemberg) (Allergo J 2015, 24(8);68-71 with kind permission of the Allergo Journal editorial board). As part of an agreement between the editorial boards of the respective journals, this paper will be published simultaneously in Allergo Journal and in Allergologie, in order to reach the widest possible German-speaking specialist readership in a timely manner.

\section{Conflict of interest}

R. Brehler has received funds for trials conducted by the UKM from ALK-Abello, Allergopharma, Bencard, HAL-Allergy, Circassia, and Novartis/Leti; for lecture activities from: ALK, Allergopharma, Bencard, Basilea, Dr. Pfleger, Hal, Hermal, MSD, MEDA, MedUpdate, Novartis, Pierre Fabre, Pohl Boskamp, Siemens, Stallergenes, Thermo Fisher; consultancy fees from: Allergopharma, Bencard, Leti, Novartis, Stallergenes; and travel subsidies from: ALK, Allergopharma, Bencard, Leti, Novartis.

L. Klimek has received funds for studies, consultant/expert activities, or remunerated collaboration on a scientific advisory committee from ALK-Abello, Denmark, Allergopharma, Germany, Boehringer Ingelheim, Germany, Lofarma, Italy,
MEDA Pharma, Germany, Novartis, Switzerland; for lecture and training activities or remunerated authorships or co-authorships from ALK-Abello, Denmark, Allergopharma, Germany, Bencard, Great Britain, Bionorica, Germany, Biomay, Austria, Cytos, Switzerland, HAL, Netherlands, Hartington, Spain, GSK, Great Britain, Leti, Spain, Lofarma, Italy, MEDA, Germany, Novartis, Switzerland, Roxall, Germany; financial donations (third-party funds) for research projects or direct financing for staff from ALK-Abello, Denmark, Allergopharma, Germany, Artu-Biologicals, Netherlands, Bencard, Great Britain, Bionorica, Germany, Biomay, Austria, Cytos, Switzerland, HAL, Netherlands, Hartington, Spain, GSK, Great Britain, Leti, Spain, Lofarma, Italy, Novartis, Switzerland, Roxall, Germany.

C. Vogelberg has received funds for trials conducted by the UKD from ALK-Abello, Novartis, Boehringer Ingelheim, Engelhard Arzneimittel GmbH \& Co KG; for lecture activities from: ALK-Abello, HAL Allergy, MSD, Novartis, Engelhard Arzneimittel GmbH \& Co KG, PARI, GlaxoSmithKline; for consultancy activities from Boehringer Ingelheim, ALK-Abello, MSD, Novartis, Engelhard Arzneimittel GmbH \& Co. KG.

T. Werfel: ALK, Allergopharma, Bencard, Stallergen. $O$. Pfaar has received research funds for his institution from Allergopharma, ALK-Abello, Stallergenes, HAL-Allergie, Artu Biologicals, Allergy Therapeutics/Bencard, Hartington, Lofarma, Novartis/Leti, GlaxoSmithKline, Essex Pharma, Cytos, Curalogic, Roxall, Biomay, Thermo Fisher, Circassia, E.U (FP-7-Health-2013-Innovation 1), Biotech Tools and MEDA-Pharma; he has received personal funds in his capacity as a consultant for Allergopharma, Anergis, Bencard, HAL Allergy, Novartis/LETI, MEDA-Pharma, ALK-Abello, Biotech Tools, GfK Bridgehead, NAVI-

GANT-consulting, Sanofi, Guidepoint Global Advisors, Pohl-Boskamp, Stallergenes, Mobile Chamber Experts (ein GA2LEN Partner); in addition, OP has received personal funds for lectures and the writing of book chapters or teaching material from ALK-Abello, Allergopharma, Stallergenes, HAL-Allergy, Allergy Therapeutics/Bencard, Hartington, Lofarma, Novartis/Leti, GlaxoSmithKline, Roxall, Thermo Fisher, MEDA-Pharma, Schattauer, Springer, and GSK; OP has received travel subsidies from HAL-Allergie, Allergopharma, EAACI, DGAKI and DGP; he is the current chairman of the Immunotherapy Interest Group (IT IG) of the EAACl, as well as secretary of the ENT department of the DGAKI.

E. Hamelmann has received personal funds in his capacity as a consultant for Allergopharma, Bencard, HAL Allergy, Novartis/LETI, ALK-Abello, Stallergenes, Boehringer Ingelheim; in addition, he has received personal funds for lectures and the writing of book chapters or teaching material from ALK-Abello, Allergopharma, Stallergenes, Allergy Therapeutics/Bencard, Novartis/Leti, and GSK; he is currently general secretary of the German Society for Allergology and Clinical Immunology (Deutsche Gesellschaft für Allergologie und klinische Immunologie, DGAKI), as well as spokesman for the department of pediatrics of the DGAKI, chairman of the department of asth$\mathrm{ma} / \mathrm{complex}$ asthma of the German Society of Pediatric Pneumology (Gesellschaft für Pädiatrische Pneumologie, GPP) and deputy spokesman of the working group for research of the German Society of Pediatric Allergology (Gesellschaft für Pädiatrische Allergologie, GPA).

\section{Cite this as}

Brehler R, Klimek L, Vogelberg C, Werfel T, Pfaar O, Hamelmann E. Evidence vs. efficacy in allergen-specific immunotherapy: Considerations using the example of tradable products in Germany. Allergo J Int 2016;25:38-43

DOI: 10.1007/s40629-016-0096-2 


\section{References}

1. Akdis M, Akdis CA. Mechanisms of allergen-specific immunotherapy: multiple suppressor factors at work in immune tolerance to allergens. J Allergy Clin Immunol 2014;133:621-31

2. Abramson MJ, Puy RM, Weiner JM. Injection allergen immunotherapy for asthma. Cochrane Database Syst Rev 2010;(8):CD001186

3. Burks AW, Calderon MA, Casale T, Cox L, Demoly P, Jutel $M$ et al. Update on allergy immunotherapy: American Academy of Allergy, Asthma \& Immunology/European Academy of Allergy and Clinical Immunology/PRACTALL consensus report. J Allergy Clin Immunol 2013;131:128896

4. Pfaar O, Bachert C, Bufe A, Buhl R, Ebner C, Eng P et al. Guideline on allergen-specific immunotherapy in IgE-mediated allergic diseases - S2k Guideline of the German Society for Allergology and Clinical Immunology (DGAKI), the Society for Pediatric Allergy and Environmental Medicine (GPA), the Medical Association of German Allergologists (AeDA), the Austrian Society for Allergy and Immunology (ÖGAl), the Swiss Society for Allergy and Immunology (SGAI), the German Society of Dermatology (DDG), the German Society of Oto-Rhino-Laryngology, Head and Neck Surgery (DGHNO-KHC), the German Society of Pediatrics and Adolescent Medicine (DGKJ), the Society for Pediatric Pneumology (GPP), the
German Respiratory Society (DGP), the German Association of ENT Surgeons (BV-HNO), the Professional Federation of Paediatricians and Youth Doctors (BVKJ), the Federal Association of Pulmonologists (BDP) and the German Dermatologists Association (BVDD). Allergo J Int 2014;23:282-319

5. Canonica GW, Baena-Cagnani CE, Bousquet J, Bousquet $\mathrm{PJ}$, Lockey RF, Malling $\mathrm{HJ}$ et al. Recommendations for standardization of clinical trials with allergen specific immunotherapy for respiratory allergy. A statement of a World Allergy Organization (WAO) taskforce. Allergy 2007;62:317-24

6. Deutsches Netzwerk Evidenzbasierte Medizin e.V., ed. www.ebm-netzwerk.de/was-ist-ebm/grundbegriffe/ definitionen

7. Pfaar O, Demoly P, Gerth van Wijk R, Bonini S, Bousquet J, Canonica GW et al; European Academy of Allergy and Clinical Immunology. Recommendations for the standardization of clinical outcomes used in allergen immunotherapy trials for allergic rhinoconjunctivitis: an EAACI Position Paper. Allergy 2014;69:854-67

8. Englert L, May S, Kaul S, Vieths S. [The therapy allergens ordinance ("Therapieallergene-Verordnung"). Background and effects]. Bundesgesundheitsbl Gesundheitsforsch Gesundheitsschutz 2012;55:351-7

9. Bachert C. Überarbeitete S2k-Leitlinie zur (allergen-)spezifischen Immuntherapie. Allergologie 2015;38:178-80 